Louisiana State University

LSU Digital Commons

Faculty Publications

Department of Physics \& Astronomy

$12-28-2001$

\title{
Tailoring harmonic radiation to different applications using a genetic algorithm
}

L. Roos

Lunds Tekniska Högskola

M. B. Gaarde

Lunds Tekniska Högskola

A. L'Huillier

Lunds Tekniska Högskola

Follow this and additional works at: https://digitalcommons.Isu.edu/physics_astronomy_pubs

\section{Recommended Citation}

Roos, L., Gaarde, M., \& L'Huillier, A. (2001). Tailoring harmonic radiation to different applications using a genetic algorithm. Journal of Physics B: Atomic, Molecular and Optical Physics, 34 (24), 5041-5054. https://doi.org/10.1088/0953-4075/34/24/307

This Article is brought to you for free and open access by the Department of Physics \& Astronomy at LSU Digital Commons. It has been accepted for inclusion in Faculty Publications by an authorized administrator of LSU Digital Commons. For more information, please contact ir@lsu.edu. 


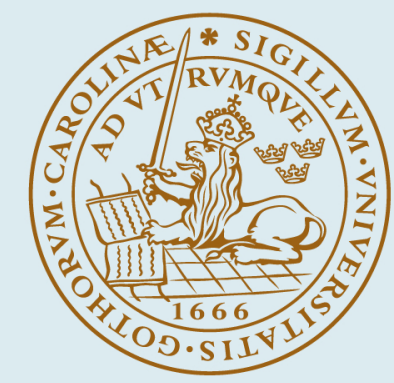

\title{
LUND UNIVERSITY
}

\section{Tailoring harmonic radiation to different applications using a genetic algorithm}

\author{
Roos, L; Gaarde, M. B; L'Huillier, Anne
}

Published in:

Journal of Physics B: Atomic, Molecular and Optical Physics

DOI:

$10.1088 / 0953-4075 / 34 / 24 / 307$

2001

Link to publication

Citation for published version (APA):

Roos, L., Gaarde, M. B., \& L'Huillier, A. (2001). Tailoring harmonic radiation to different applications using a genetic algorithm. Journal of Physics B: Atomic, Molecular and Optical Physics, 34(24), 5041-5054.

https://doi.org/10.1088/0953-4075/34/24/307

\section{Total number of authors:}

3

\section{General rights}

Unless other specific re-use rights are stated the following general rights apply:

Copyright and moral rights for the publications made accessible in the public portal are retained by the authors

and/or other copyright owners and it is a condition of accessing publications that users recognise and abide by the

legal requirements associated with these rights.

- Users may download and print one copy of any publication from the public portal for the purpose of private study

or research.

- You may not further distribute the material or use it for any profit-making activity or commercial gain

- You may freely distribute the URL identifying the publication in the public portal

Read more about Creative commons licenses: https://creativecommons.org/licenses/

Take down policy

If you believe that this document breaches copyright please contact us providing details, and we will remove

access to the work immediately and investigate your claim. 
Tailoring harmonic radiation to different applications using a genetic algorithm

This article has been downloaded from IOPscience. Please scroll down to see the full text article.

2001 J. Phys. B: At. Mol. Opt. Phys. 345041

(http://iopscience.iop.org/0953-4075/34/24/307)

View the table of contents for this issue, or go to the journal homepage for more

Download details:

IP Address: 130.235.188.104

The article was downloaded on 05/07/2011 at 07:11

Please note that terms and conditions apply. 


\title{
Tailoring harmonic radiation to different applications using a genetic algorithm
}

\author{
L Roos ${ }^{1}$, M B Gaarde ${ }^{1,2}$ and A L'Huillier ${ }^{1}$ \\ ${ }^{1}$ Department of Physics, Lund Institute of Technology, PO Box 118, S-221 00 Lund, Sweden \\ 2 Department of Physics and Astronomy, Louisiana State University, Baton Rouge, \\ LA 70803-4001, USA
}

Received 7 August 2001, in final form 18 October 2001

Published 10 December 2001

Online at stacks.iop.org/JPhysB/34/5041

\begin{abstract}
We use a genetic algorithm to theoretically optimize several properties of extreme ultraviolet (XUV) radiation, generated as high-order harmonics in xenon and argon. We maximize the harmonic pulse energy, minimize the pulse duration or optimize the temporal coherence by varying at the same time two or three parameters that are easily accessible in experiments, related to the characteristics of the laser beam and the nonlinear medium. For the 15th and 29th harmonics in argon, we find up to $10^{9}$ photons per pulse, and pulse durations as short as $6 \mathrm{fs}$ generated by a $50 \mathrm{fs}$ laser pulse. We can also tailor the phase matching conditions to spectrally select the transform-limited part of the harmonic radiation. This allows us to identify conditions when the time structure of the XUV radiation presents a train of attosecond pulses. We find that the optimum conditions for the different properties are in general not the same. They depend in particular on whether the harmonic belongs to the plateau or the cutoff region of the harmonic spectrum. This reflects the unavoidable interplay between the microscopic intensity-dependent harmonic phase and the macroscopic phase matching conditions imposed by a nonlinear medium interacting with an intense, focused laser beam.
\end{abstract}

(Some figures in this article are in colour only in the electronic version)

\section{Introduction}

In the interaction between an intense ultrafast laser pulse and an atomic gas, very high-order odd harmonics of the fundamental laser frequency can be generated. In this highly nonperturbative process, near-infrared laser light is converted into radiation with photon energy up to several hundred eV [1,2]. The generated harmonics are coherent, directional and of shorter duration than the driving laser pulse. These properties are used in the many applications of the harmonic radiation that have appeared, for example pump-probe lifetime measurements [3], time-resolved surface physics [4] and extreme ultraviolet (XUV) interferometry [5]. Recently, 
it has also been demonstrated that the harmonic radiation is intense enough to drive nonlinear processes (two- or four-photon ionization) [6,7]. Finally, harmonic generation is a promising source for the production of sub-femtosecond, so-called attosecond, pulses. Experimental support for the idea that a set of high-order harmonics can produce a regular train of attosecond pulses has already been presented [8].

The increasing use of harmonics as an XUV light source sets new demands both on the characterization and the optimization of the radiation. Different applications have different requirements for the properties of the harmonic radiation. Although most users clearly benefit from a high pulse energy, some applications additionally require a large photon energy, a short pulse duration, good spatial coherence properties and/or a good temporal coherence (narrow spectrum). To find the optimal conditions for a given application is not an easy task: numerous studies have demonstrated the importance of both the laser characteristics (such as intensity [9], frequency [10], phase [11], pulse duration and shape [12,13] and focusing conditions [14]) and the nonlinear medium (species [15], atomic density [16,17] and length of the medium [18]) to the harmonic properties. Some of these parameters influence the microscopic part of the interaction between the atoms and the laser field; others affect the macroscopic propagation and phase matching conditions. The detailed characteristics of the harmonic radiation depend in general on the interplay between these two parts. A complete optimization is therefore a true multidimensional problem. All experimental and theoretical studies performed so far, however, have optimized a specific property of the harmonic radiation with respect to only one of the above-mentioned parameters.

We present here a theoretical study of optimization of several features of high-harmonic generation in argon and xenon using a genetic algorithm (GA). Genetic and other types of evolutionary algorithms are feedback controlled search algorithms that are useful when optimizing complicated functions of multiple parameters, which can have several local maxima and minima ${ }^{3}[19,20]$. These search methods have borrowed their features from nature, mimicking the mechanisms of evolution, and have been used extensively, for instance, in adaptive optics control [21]. Recently, an evolutionary algorithm was applied to optimize the yield of a single harmonic in argon via pulse shaping [13,22]. We apply this algorithm to three problems.

(1) The optimization of the pulse energy of one harmonic (the ninth harmonic in xenon; the 15th and 29th harmonics in argon).

(2) The minimization of the pulse duration of one harmonic (the 15th and 29th harmonics in argon), while keeping a high pulse energy. This kind of optimization is of interest for pump-probe-type applications, which require high temporal resolution, as well as for applications in multiphoton physics, requiring a high intensity.

(3) The optimization of the temporal coherence of the 15th and 19th harmonics in argon. Temporal coherence is important for instance in spectroscopy. In addition, as described in detail in [23-25], a narrow, almost transform-limited, harmonic spectrum implies that among the many quantum path contributions to the harmonic dipole moment, only one is dominating. If this is also true for a few neighbouring harmonics, the time structure of the sum of these harmonics presents a train of attosecond pulses [26].

We optimize these properties with respect to two or three parameters, among the pressure and length of the nonlinear medium, the intensity of the driving field and the position of the laser focus relative to the centre of the medium. These parameters are all easy to access in most experiments. Our calculations of the harmonic field are three dimensional, and include both the single-atom response and the results of propagation through the macroscopic medium.

3 Many useful answers to questions about GAs can also be found at http://surf.de.uu.net/encore/www/ 
We first briefly describe our theoretical method in section 2. In section 3 we present the results of the optimization of harmonic pulse energy in xenon and argon, and of the minimization of the harmonic pulse duration in argon. Finally, we describe in section 4 how the GA is used to optimize the temporal coherence of the 15th harmonic in argon, and the sub-cycle time structure of the combination of harmonics 15-23. A short summary is given in section 5 .

\section{Method}

Our calculation contains three different parts: the single-atom response, the macroscopic response and the optimization performed by the GA.

We start by calculating the response from a single atom to an intense laser field with a quasi-constant intensity. This calculation is performed for many different intensities, to obtain the intensity-dependent dipole moments $d_{q}(I)$ for each harmonic $q$. In the case of xenon, these dipole moments are calculated within the semi-classical model described in [27]. Note that this approximation is quite crude for the ninth harmonic, which is only just above the ionization threshold in xenon. However, as we demonstrate below, the conditions for generation of the ninth harmonic are dominated by absorption in the xenon gas jet, and an approximate description of the dipole moment is therefore sufficient. The argon singleatom data are calculated by numerical integration of the three-dimensional time-dependent Schrödinger equation within the single-active-electron approximation (see for instance [28]).

The macroscopic harmonic field is calculated by solving the Maxwell wave equation within the paraxial and the slowly varying envelope approximations [29]. We use the atomic dipole moments $d_{q}$ as a source of the nonlinear part of the polarization field $P_{q}^{\mathrm{NL}}(\boldsymbol{r}, t)=$ $N_{\mathrm{a}}(\boldsymbol{r}, t) d_{q}(I(\boldsymbol{r}, t))$, where $N_{\mathrm{a}}(\boldsymbol{r}, t)$ is the density of atoms in the gas jet, and $I(\boldsymbol{r}, t)$ represents the intensity distribution of the focused laser beam. We include ionization as a slowly varying depletion of the neutral medium, using ionization rates calculated as described in [30]. The free electrons thereby released contribute to the refractive index of the nonlinear medium and cause the driving laser beam to defocus. The calculation also includes dispersion and absorption caused by the neutral atoms in the gas jet. We are thus able to accurately model the phase matching effects arising from the combination of the single-atom dipole phase (which depends on the intensity of the laser field [27]), the focusing of the laser beam and the ionization of the medium.

The propagation calculations are run within the framework of the GA, which searches for the particular combination of the macroscopic parameters that gives the optimum result. The GA works in the following way [31]: the boundaries within which the optimum is sought are set for each parameter, which can take on 64 different values within its boundaries. When optimizing in two dimensions, for instance, there are $64 \times 64$ combinations of parameters, or possible solutions to the optimization problem. The GA represents each possible solution, or individual, with a string of bits, termed a chromosome. For example the possible solution 'a medium length of $3 \mathrm{~mm}$ and a pressure of 10 Torr' is represented as [010110]. (For the sake of clarity we assume here that each parameter can only take $2^{3}=8$ values in this example.)

The first generation of individuals is selected randomly. Typically, we use a population size of 50. For each generation, the following steps are then carried out: (i) all the individuals are evaluated and assigned a fitness value. In our case this means we calculate the macroscopic harmonic field resulting from each configuration, and attribute a fitness value depending on the harmonic property we are interested in. The next generation of individuals is chosen by applying the three GA operators selection, mutation and crossover. (ii) The selection operator chooses which of the individuals from the present generation will be transferred to 
the next generation. The individuals are ranked according to their fitness, and then selected randomly with a certain probability based on the fitness. A very fit individual thus receives a high probability and can be selected several times, while a low-fitness individual may not be selected at all. (iii) The mutation operator, which is used very sparsely, selects a few individuals and replaces one (randomly selected) bit in a chromosome by randomly 0 or 1 (e.g. creating the chromosome [010101] from [110101]). (iv) The crossover operator takes two individuals at a time and exchanges part of their chromosomes. For example the two chromosomes [010101] and [111000] can create the chromosomes [010000] and [111101]. This is done for $60 \%$ of the selected solutions. The use of the mutation and the crossover operators ensures that the GA does not become trapped in a local optimum. The fittest individual, however, always survives to the next generation unchanged. This is called elitism. The steps (i)-(iv) are then repeated until no new possible solutions appear between two consecutive generations. In the optimizations presented in this work, the GA typically converges after 30-60 iteration steps. Although the population size does not change between iteration steps, the number of new individuals that actually need to be evaluated decreases since many individuals reappear. Our propagation code therefore initially checks whether it already knows the fitness value of an individual before the calculation is performed. This reduces the time to reach convergence, which typically takes 200-400 evaluations for a two-dimensional optimization, and around 400-700 for optimizations in three dimensions.

\section{Results}

\subsection{Optimization of pulse energy}

3.1.1. Xenon. We start by optimizing the number of photons generated in xenon in the ninth harmonic as a function of the pressure, $P$, and the length, $L$, of the medium. We use driving laser pulses with a wavelength of $800 \mathrm{~nm}$, and a duration of $50 \mathrm{fs}$. The laser beam is focused in the centre of the gas jet to a peak intensity of $I_{0}=9 \times 10^{13} \mathrm{~W} \mathrm{~cm}^{-2}$. We mimic realistic experimental conditions by using a truncated, loosely focused Gaussian beam. Numerically, this beam is calculated as the Hankel transform of a $4 \mathrm{~cm}$ diameter Gaussian beam which has been apertured down to a diameter of $1.5 \mathrm{~cm}$. The confocal parameter $b$ of the resulting beam is approximately $10.5 \mathrm{~cm}$.

In figure 1 we plot the results of all the evaluations of harmonic pulse energy that the GA has performed. A high harmonic pulse energy (measured in the number of photons produced in the pulse) is represented with a green symbol and a low pulse energy with a dark/red symbol, according to the colour bar. Close to the optimum value(s) the density of points is higher than elsewhere, reflecting how the GA evolves. This allows us to obtain information about the behaviour of the function close to its optimum value.

It is evident from the figure that the maximum harmonic pulse energy that can be obtained for any given pressure is fairly constant. For low pressures the optimum occurs at long medium lengths, and for higher pressures at shorter medium lengths. This behaviour can be attributed to absorption of the ninth harmonic in xenon, for which the cross-section is relatively high.

To illustrate this, we calculate the absorption length $L_{\text {abs }}$ for the ninth harmonic in xenon, using the one-photon absorption cross section $\sigma$ and the atomic density $N_{\mathrm{a}}, L_{\mathrm{abs}}=\frac{1}{\sigma N_{\mathrm{a}}} . L_{\mathrm{abs}}$ is the length over which the power of the harmonic has decreased by a factor of $e$. The solid curve in figure 1, which closely follows the optimum medium length for a given pressure, corresponds to $L=3 L_{\text {abs }}$. That the optimum medium length is so much longer than the absorption length reflects that the harmonic is both generated and absorbed as it propagates through the medium. The optimum conditions for the generation of this harmonic are thus mostly determined by absorption [17]. 


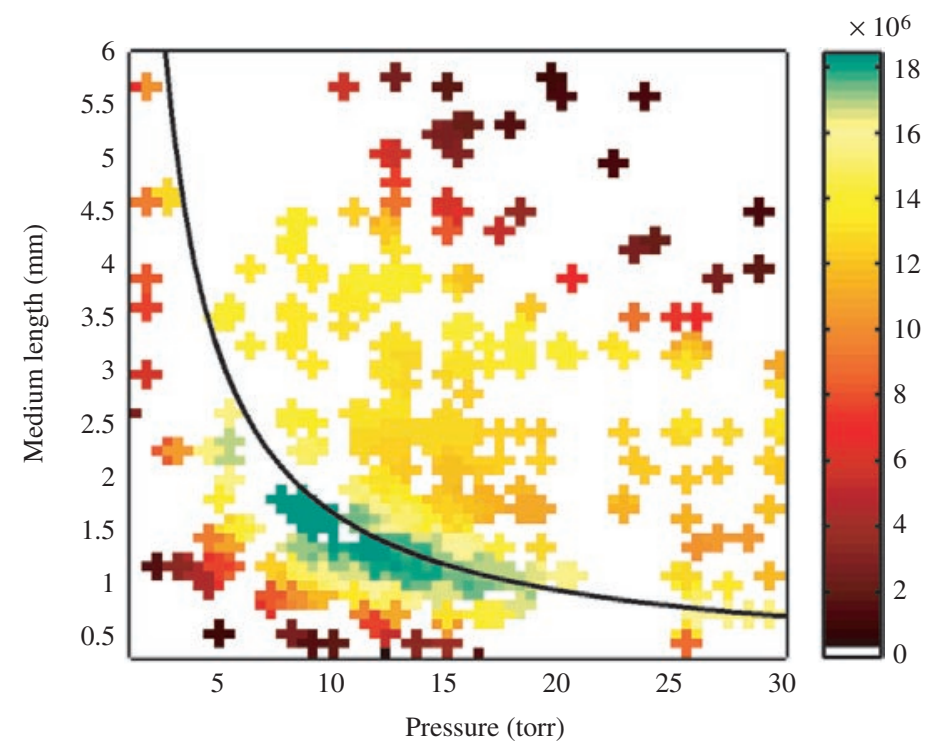

Figure 1. Optimization of the number of photons in the ninth harmonic in xenon with respect to pressure and medium length. The number of photons is given in accordance with the colour bar on the right.

Table 1. Optimizations of pulse energy in $\mathrm{H} 15$ and $\mathrm{H} 29$ in argon with respect to medium length, $L$, and focus position relative to medium, $Z_{0}$. We show the parameters that lead to the maximum number of photons. We also list the boundaries of the region within which the number of photons has not decreased by more than a factor of two from the optimum value. The optimizations were performed with different laser intensities and focusing conditions as summarized in this table.

\begin{tabular}{|c|c|c|c|c|c|c|c|c|c|}
\hline \multirow[b]{3}{*}{ Harmonic order } & \multicolumn{6}{|c|}{$b \approx 10.5 \mathrm{~cm}$} & \multicolumn{3}{|c|}{$b=1 \mathrm{~cm}$} \\
\hline & \multicolumn{2}{|c|}{$2.4 \times 10^{14}$} & \multirow[t]{2}{*}{$\mathrm{W} \mathrm{cm}^{-2}$} & \multicolumn{2}{|c|}{$1.5 \times 10^{14}$} & \multirow[t]{2}{*}{$\mathrm{W} \mathrm{cm} \mathrm{cm}^{-2}$} & \multicolumn{2}{|c|}{$2.4 \times 10^{14}$} & \multirow[t]{2}{*}{$\mathrm{W} \mathrm{cm}^{-2}$} \\
\hline & 15 & 29 & & 15 & 29 & & 15 & 29 & \\
\hline Optimum $L(\mathrm{~mm})$ & 3 & 4 & & 1 & 1.3 & & 2 & 5 & \\
\hline Optimum $Z_{0}(\mathrm{~mm})$ & 30 & 20 & & 5 & 1 & & 2 & 0.9 & \\
\hline Optimum region $L(\mathrm{~mm})$ & $0.5-4$ & $3-5$ & & $0.5-3$ & $0.8-3$ & & $0.1-4$ & $2-7$ & \\
\hline Optimum region $Z_{0}(\mathrm{~mm})$ & $-30-30$ & $-30-30$ & & $-20-20$ & $-12-1$ & & $1.5-3.5$ & -0.6 & $5-2$ \\
\hline Photon number $\left(\times 10^{8}\right)$ & 7 & 16 & & 3 & 1 & & 0.5 & 1 & \\
\hline Figure & $2(a)$ & $2(b)$ & & - & - & & $2(c)$ & $2(d$ & \\
\hline
\end{tabular}

3.1.2. Argon. We now turn to the optimization of the pulse energy of the 15 th and the 29th harmonics generated in argon. We keep the peak intensity fixed at two different values close to the saturation intensity, and optimize with respect to $P, L$ and the relative position of the laser focus to the centre of the gas jet $Z_{0}$. The laser wavelength is $810 \mathrm{~nm}$ and the pulse duration 50 fs.

First we optimize these harmonics with respect to $L$ and $Z_{0}$, with a pressure of 50 Torr. We present six different optimizations. For both harmonics we try in turn the following configurations: (i) high intensity and loose focusing, (ii) low intensity and loose focusing and (iii) high intensity and tight focusing. A summary of the optimizations and their results is presented in table 1 . 

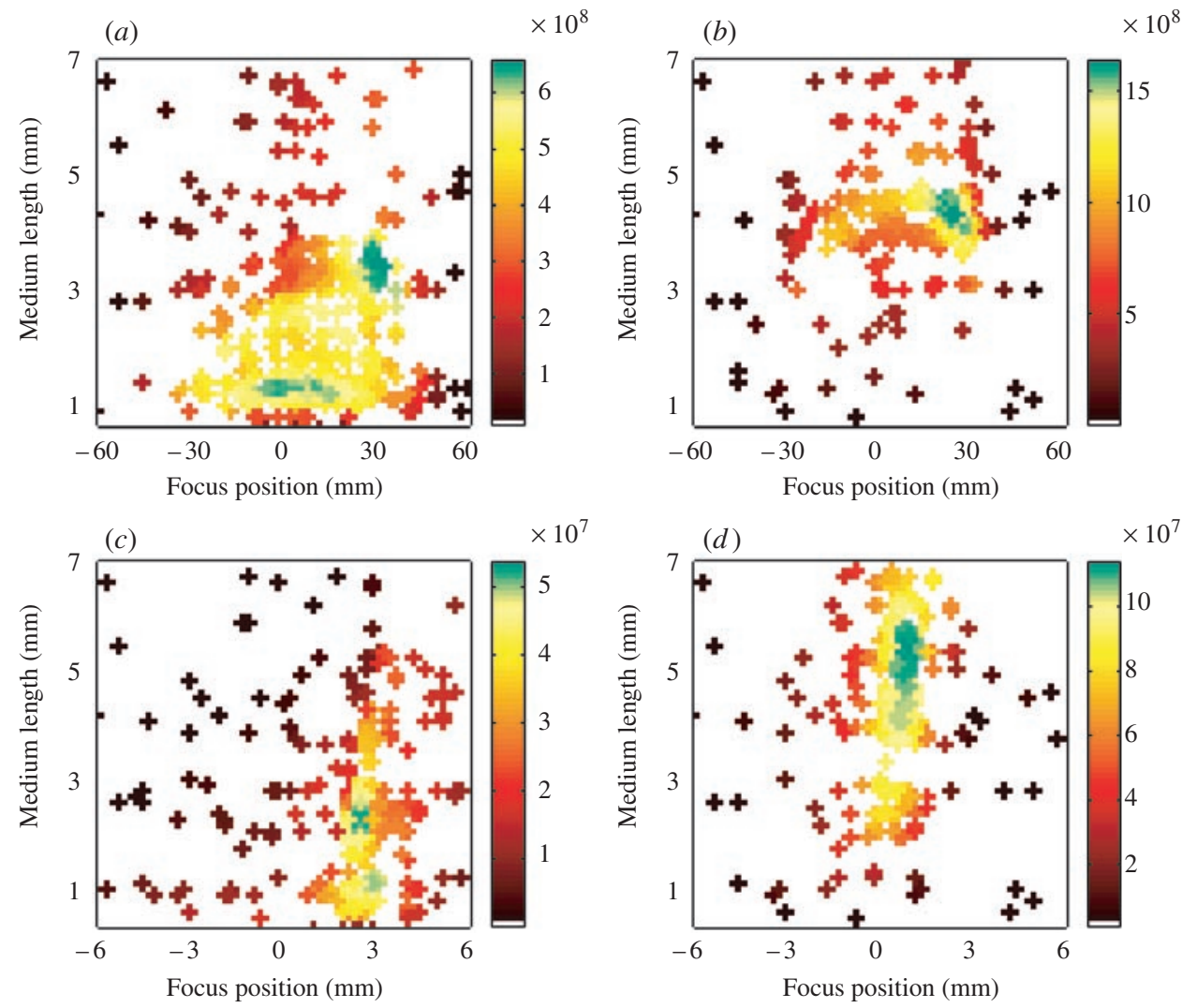

Figure 2. Optimization of the number of photons with respect to focus position and medium length in argon. The laser peak intensity is $I_{0}=2.4 \times 10^{14} \mathrm{~W} \mathrm{~cm}^{-2}$. (a) $\mathrm{H} 15, b \approx 10.5 \mathrm{~cm}$. (b) $\mathrm{H} 29$, $b \approx 10.5 \mathrm{~cm}$. (c) $\mathrm{H} 15, b=1 \mathrm{~cm}$. (d) $\mathrm{H} 29, b=1 \mathrm{~cm}$. At $Z_{0}=0$ the laser focus is in the centre of the medium, $Z_{0}<0$ means that the focus is before the centre of the medium, while the focus is after the centre of the gas medium for $Z_{0}>0$.

(i) We first compare the optimizations of the 15th and the 29th harmonic, presented in figures $2(a)$ and $(b)$, respectively, using a laser intensity of $I_{0}=2.4 \times 10^{14} \mathrm{~W} \mathrm{~cm}^{-2}$ and the same focusing as in the previous section ${ }^{4}, b \approx 10.5 \mathrm{~cm}$. The highest number of photons per pulse is close to $10^{9}$ for both harmonics. We define an 'optimum region' in which the number of photons has not decreased by more than approximately a factor of two from the optimum value (see table 1). As evident from figure 2(a) and table 1, the optimum region for the 15th harmonic is quite large. The corresponding result for the 29th harmonic shows an optimum region that is smaller in $L$, but similar in $Z_{0}$. The optimum configuration is different for the two harmonics, even though there is a small overlap between the optimum regions.

(ii) Next we compare the results obtained at the two intensities, $I_{0}=2.4 \times 10^{14} \mathrm{~W} \mathrm{~cm}^{-2}$ (figures 2(a) and $(b)$ ) and $I_{0}=1.5 \times 10^{14} \mathrm{~W} \mathrm{~cm}^{-2}$ (not shown in figure 2). We present these results in table 1 . The optimal $L$ found with the low intensity is substantially shorter than that found with the high intensity, and the optimal position of the focus is closer to the centre

$4 \quad I_{0}$ is the intensity at the peak of the laser pulse and in the centre of the laser focus. When $Z_{0}$ is different from zero, the intensity in the centre of the medium is thus lower. In addition, defocusing caused by the presence of free electrons often lowers the actual peak intensity. 
of the gas medium. In addition, the optimum region is smaller. For the 15 th harmonic there is an overlap between the optimum regions for the two intensities. In contrast, there is no overlap for the 29th harmonic, indicating that phase matching conditions are different at the two intensities. In addition, the maximum number of photons obtained at the two intensities is almost the same for the 15th harmonic, but differs by more than an order of magnitude for the 29th harmonic. This behaviour is rather general: phase matching conditions strongly depend on whether the harmonic belongs to the plateau or the cutoff region of the harmonic spectrum. In our example, the 15th harmonic belongs to the plateau for both intensities, whereas the 29th harmonic is in the plateau at $2.4 \times 10^{14} \mathrm{~W} \mathrm{~cm}^{-2}$ and in the cutoff at $1.5 \times 10^{14} \mathrm{~W} \mathrm{~cm}^{-2}$.

(iii) Here we optimize the two harmonics using a tighter focusing geometry. We use a Gaussian beam with a confocal parameter of $1 \mathrm{~cm}$, and a peak intensity of $2.4 \times 10^{14} \mathrm{~W} \mathrm{~cm}^{-2}$. The results are shown in figures $2(c)(\mathrm{H} 15)$ and $(d)(\mathrm{H} 29)$ and table 1. Since the phase matching conditions have changed a lot from the loose focusing geometry, the results of figures $2(c)$ and $(d)$ are very different from those of 2(a) and $(b)$. Both harmonics are optimized when the laser focus is located after the medium, in agreement with previous results [14]. However, the 29 th harmonic is optimized with substantially longer medium than the 15th harmonic; there is only a small overlap between the optimum regions of the two harmonics. The configuration that gives the highest pulse energy in the 29th harmonic is actually quite bad for the 15 th.

Finally, we optimize the pulse energy of the 15 th and the 29th harmonics with respect to three parameters, $L, Z_{0}$ and $P$. We use the loose focusing geometry, $b \approx 10.5 \mathrm{~cm}$ with a peak intensity of $2.4 \times 10^{14} \mathrm{~W} \mathrm{~cm}^{-2}$. For the 29th harmonic, the maximum obtained pulse energy is a factor of two higher than the results obtained at 50 Torr. The corresponding gain for the 15 th harmonic is only a factor of 1.2. The $P$ that gives the highest pulse energy is near 200 Torr for both harmonics, while $L$ is shorter and $Z_{0}$ similar. Even though absorption of the 15 th harmonic in argon is not much smaller than that of the ninth harmonic in xenon, these results indicate that the generation of the 15th harmonic in argon is not primarily limited by absorption. In comparison, the absorption of the 29th harmonic in argon is very small.

We also perform optimizations of the adjacent harmonic orders in the same conditions as above. The results obtained with the 31 st harmonic correspond well to those found for the 29 th harmonic, and, likewise, the 15 th and the 17 th harmonics behave similarly to each other. We therefore believe that the results described above for 15th and 29th harmonics could be generalized to represent a typical plateau and cutoff harmonic, respectively.

In conclusion, depending on the selected combination of harmonic order, peak intensity and focusing geometry, the optimum configuration can vary substantially. Since the optimum for each harmonic is a delicate balance between intensity, ionization, phase matching and absorption, a change of the fixed values requires a whole new optimization in most cases. An optimization algorithm coupled to an experiment could therefore be tremendously useful, either in predicting optimal conditions or actually finding them in practice.

\subsection{Optimization of pulse duration}

We now use the GA to minimize the harmonic pulse duration in the following way. The time profile $I_{q}(t)$ of the harmonic pulse is calculated by radial integration of the time-dependent radial profile $I_{q}(r, t)$ that is the harmonic intensity at the output of the medium. Then, a Gaussian function is fitted to $I_{q}(t)$. The full width at half maximum (FWHM) of the Gaussian fit is used as a measure of the pulse duration. In some cases this approximation is quite crude as the harmonic time profiles can be complex and for instance have multiple peaks.

We minimize the pulse durations of the 15th and 29th harmonics in argon in the same conditions as in section 3.1.2. We start by optimizing with respect to $L$ and $Z_{0}$, keeping a 
Table 2. Optimizations of pulse durations in $\mathrm{H} 15$ and $\mathrm{H} 29$ in argon with respect to $L$ and $Z_{0}$

\begin{tabular}{|c|c|c|c|c|c|c|c|c|}
\hline \multirow[b]{3}{*}{ Harmonic order } & \multicolumn{5}{|c|}{$b \approx 10.5 \mathrm{~cm}$} & \multicolumn{3}{|c|}{$b=1 \mathrm{~cm}$} \\
\hline & $2.4 \times 10^{14}$ & \multirow{2}{*}{$\mathrm{W} \mathrm{cm}^{-2}$} & \multicolumn{2}{|c|}{$1.5 \times 10^{14}$} & \multirow[t]{2}{*}{$\mathrm{W} \mathrm{cm}^{-2}$} & \multicolumn{2}{|c|}{$2.4 \times 10^{14}$} & \multirow[t]{2}{*}{$\mathrm{W} \mathrm{cm}-2$} \\
\hline & $15 \quad 29$ & & 15 & 29 & & 15 & 29 & \\
\hline Pulse duration (fs) & 13 & & 12 & 9 & & 8 & 13 & \\
\hline Photon number $\left(\times 10^{8}\right)$ & 4 & & 0.7 & 0.3 & & 0.2 & 0.8 & \\
\hline
\end{tabular}

constant pressure of 50 Torr. To ensure that we find a short pulse that also contains a substantial amount of energy, we select the boundaries of the optimization so that the number of photons in the pulse has not decreased by more than a factor of approximately ten from the maximum number of photons (obtained from the optimizations listed in table 1). In table 2 we summarize the results, showing the shortest pulse duration that was found in each case together with the corresponding number of photons. A few things can be noted. (i) The obtained pulse durations are rather short; it is possible to obtain 8 fs pulses starting from a 50 fs long fundamental pulse. (ii) The number of photons contained in these pulses is still high; typically it has been reduced by a factor of between two and three from the maximum. This means that the power of these harmonics is high, 0.9 MW in the 29th harmonic using the high-intensity and loose-focusing conditions, for instance.

For comparison, the average pulse duration of the 50 first randomly selected $Z_{0}$ and $L$ values is typically around $20 \mathrm{fs}$, while the longest harmonic pulse durations are around $40 \mathrm{fs}$. Figure 3(a) presents two typical randomly selected time profiles of the 15th (solid curve) and the 29th harmonic (dashed curve) before optimization, both in the configuration with $I_{0}=2.4 \times 10^{14} \mathrm{~W} \mathrm{~cm}^{-2}$ and $b \approx 10.5 \mathrm{~cm}$. In figure $3(b)$ we show how the time profiles have been improved after optimization. The shortest obtained time profile of the 15 th harmonic has a duration of $13 \mathrm{fs}$, and that of the 29th harmonic is $8 \mathrm{fs}$ long. The 29th harmonic pulse has a less complicated temporal profile and is better fitted to a Gaussian shape than the 15th harmonic. The peak of both harmonic pulses is reached during the leading edge of the laser pulse (at negative times), indicating the depletion of the neutral medium caused by ionization. This effect is stronger for the 15th than for the 29th harmonic, since the latter belongs to the cutoff region and is therefore generated closer to the peak of the laser pulse.

Even more pronounced in this optimization than in the optimization of pulse energy is that the values of $Z_{0}$ and $L$ for which the shortest pulse is obtained are strongly dependent on the selected configuration. The temporal profile variation is more complex than that of the pulse energy and exhibits several local minima. This reflects the fact that the pulse duration is determined by a combination of many factors such as laser intensity, ionization and phase matching conditions.

We optimize also with respect to three variables, $Z_{0}, L$ and $P$ at the same time, using the high intensity and long confocal parameter. It is then possible to decrease the pulse duration further. We have found pulses down to 6 fs for the 29th harmonic and just above 6 fs for the 15 th harmonic. These pulses contain a high number of photons, above $10^{9}$ for the 29 th harmonic (at around 150 Torr) and above $10^{8}$ for the 15 th harmonic (at around 170 Torr). Figure 3(c) shows the corresponding temporal profiles. It is again a strong depletion of the medium which, in combination with phase matching conditions, gives rise to the very asymmetric 15 th harmonic pulse with a sharp feature and a broad pedestal. A nonlinear process driven by the harmonic pulse will be very sensitive to a sharp, narrow feature and much less to the pedestal of the pulse. 


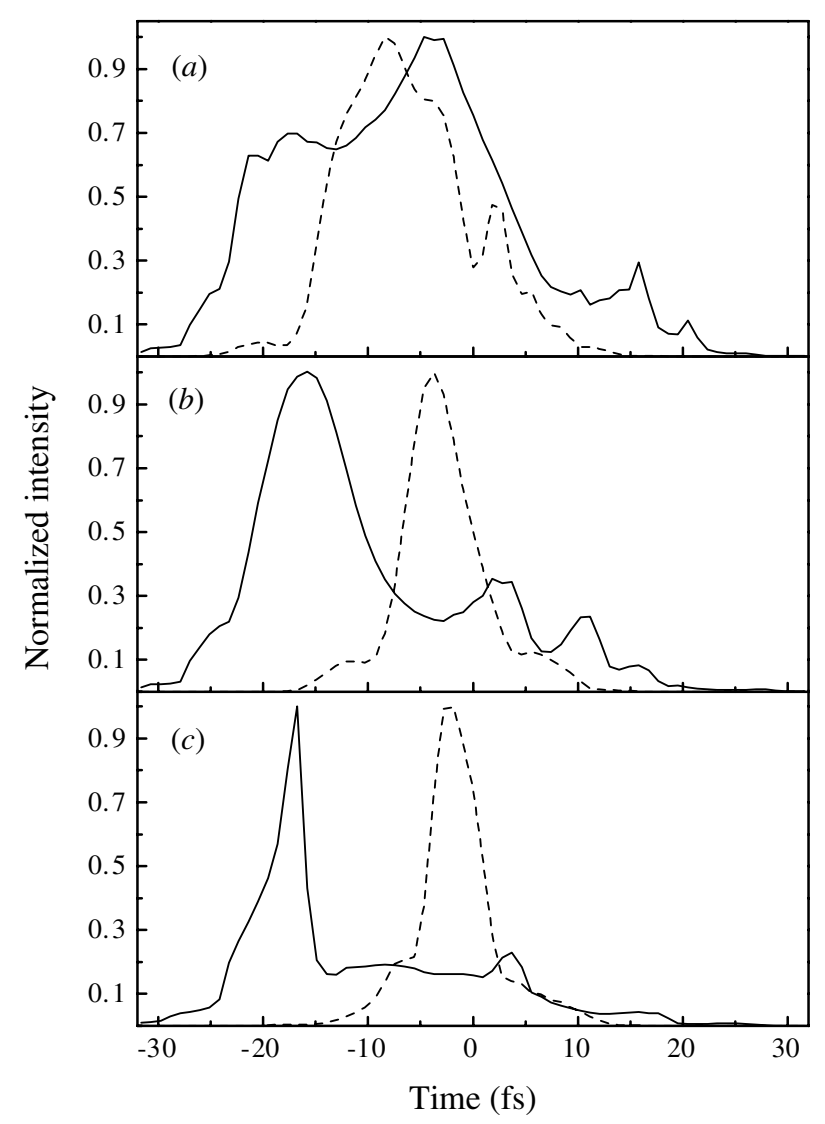

Figure 3. Temporal profiles of the 15th (solid curve) and the 29th harmonic (dashed curve). (a) Before optimization. (b) After optimization with respect to $L$ and $Z_{0}$, with a fixed pressure of 50 Torr. (c) After optimization with respect to $L, Z_{0}$ and $P$.

\section{Optimization of the harmonic coherence properties}

We finally turn to the optimization of the spectral properties of the harmonic radiation, and thereby its temporal coherence. As described in detail in $[23,24]$, the plateau harmonics in argon can each separate in the spatial and spectral domains into a well collimated part, which has a narrow spectrum, and a strongly divergent part, with a broad spectral profile. The separation is a direct result of the dynamics of the ionizing atom during the harmonic generation process. The single-atom dipole moment for each harmonic can be described as a sum of several quantum path contributions, each with a different phase behaviour [32]. The first one, often labelled $\tau_{1}$, has a phase that varies slowly as a function of intensity, while all the other contributions have much more rapid phase behaviours. In the time domain these contributions give rise to interferences, but their different phase behaviours during the course of the driving laser pulse cause them to spectrally separate. In figure 4, solid curve, we present a typical spectrum of the 15th harmonic in argon, which clearly shows this behaviour. The narrow central peak corresponds to the $\tau_{1}$-contribution, while the broad structure results from the other contributions. 


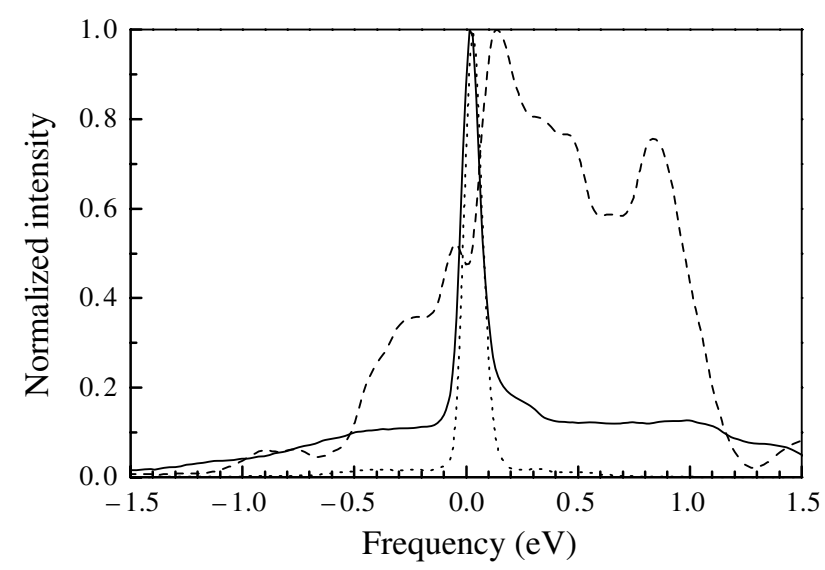

Figure 4. Spectra of the 15th harmonic in argon. In the solid curve, a typical spectrum showing a narrow $\tau_{1}$-peak and a broad pedestal. In the dashed curve, a spectrum with no significant $\tau_{1}$-peak. In the dotted curve, the spectrum obtained after optimization with respect to $I_{0}, Z_{0}$ and $L$.

In the following, we use the GA to select the well behaved $\tau_{1}$ part of the 15 th harmonic radiation, by searching for spectra where most of the energy is in the narrow central part. We are thus using the macroscopic parameters from above to select a particular microscopic harmonic behaviour, by tailoring the phase matching conditions to favour the $\tau_{1}$ contribution.

We define a fitness function which rewards spectra with a large relative $\tau_{1}$ content by calculating the spectral ratio between the $\tau_{1}$-portion of the spectrum and the whole spectrum. This is done by first calculating the spectrum from the propagated harmonic field. To locate the $\tau_{1}$ peak we find the position of the maximum amplitude in a small interval around the central frequency [24]. We then integrate the part of the spectrum that is within $0.07 \mathrm{eV}$ from the maximum, and take the ratio to the whole integrated spectrum. The value $0.07 \mathrm{eV}$ corresponds approximately to a typical half width at half maximum (HWHM) of the narrow $\left(\tau_{1}\right)$ part of the spectrum. For comparison, a Gaussian profile with HWHM of $0.07 \mathrm{eV}$ would have a spectral ratio of 0.76 . This method efficiently sorts out spectra with the largest part of their energy in the $\tau_{1}$-peak from spectra with very small $\tau_{1}$-contributions. In the latter case, the maximum spectral amplitude may not be found close to the central frequency. However, such a spectrum is of no interest to us since it has no dominant $\tau_{1}$-peak, and due to its resulting small spectral ratio it will not be favoured by the GA. An example of a spectrum without a significant $\tau_{1}$-peak is shown with the dashed curve in figure 4 . This spectrum is calculated with the parameters corresponding to the maximum number of photons in the 15 th harmonic found in section 3.1.2. The variation in the spectral shape of the 15th harmonic in different configurations is significant.

We optimize the spectral ratio as a function of the three parameters $I_{0}, Z_{0}$ and $L$. We use a pressure of 20 Torr and loose-focusing conditions, in this case a Gaussian laser beam with a confocal parameter of $b=10.5 \mathrm{~cm}$. The maximum spectral ratio that can be obtained in these conditions is 0.77 , which is obtained when $Z_{0}$ is $-31 \mathrm{~mm}$ (focusing the laser $31 \mathrm{~mm}$ before the centre of the gas jet), $L$ is $7 \mathrm{~mm}$ and the peak intensity $1.7 \times 10^{14} \mathrm{~W} \mathrm{~cm}^{-2}$. We show the corresponding spectral profile in figure 4 (dotted curve). Compared with the non-optimized spectra, the pedestal has been almost entirely suppressed. The FWHM of the narrow part of the spectrum is $0.096 \mathrm{eV}$, which corresponds to a coherence time of $19 \mathrm{fs}$. The pulse length of the 15th harmonic in this configuration is $24 \mathrm{fs}$, and the harmonic is thus very close to transform limited. 


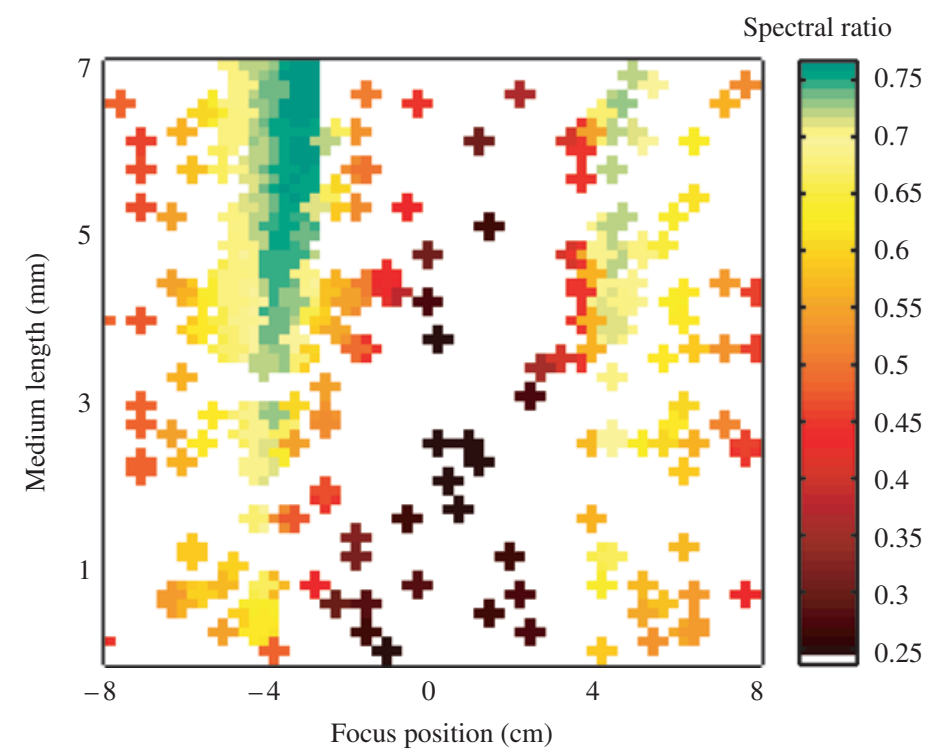

Figure 5. Optimization of the temporal coherence (spectral ratio) of the 15th harmonic in argon with respect to $L$ and $Z_{0}$, at a fixed peak intensity of $1.6 \times 10^{14} \mathrm{~W} \mathrm{~cm}^{-2}$.

At each fixed intensity there are two regions in $Z_{0}$ where the spectral ratio is close to 0.7 , one on either side of the centre of the gas jet. This is illustrated in figure 5, where we show an optimization of the spectral ratio of the 15th harmonic with respect to only $L$ and $Z_{0}$, at a fixed intensity of $I_{0}=1.7 \times 10^{14} \mathrm{~W} \mathrm{~cm}^{-2}$. Almost independently of the length of the medium, it is possible to obtain $\tau_{1}$-dominated harmonics by focusing either $3 \mathrm{~cm}$ before or $4.5 \mathrm{~cm}$ after the centre of the gas jet. Using a higher fixed intensity these regions move to larger $\left|Z_{0}\right|$ on both sides, and at a lower intensity to smaller $\left|Z_{0}\right|$. Previous (theoretical) studies have demonstrated that phase matching conditions are often favourable for the $\tau_{1}$ contribution when the laser focus is before the centre of the gas jet [26]. We here find that the $\tau_{1}$ contribution is also well phase matched at positive $Z_{0}$.

The pulse energy and the temporal coherence are-not surprisingly-not optimized in the same configuration. The number of photons obtained in the configuration optimizing the temporal coherence is $5 \times 10^{7}$ and the pulse duration is $24 \mathrm{fs}$. Compared with the maximum harmonic pulse energy or the shortest pulse duration, one thus loses a factor of ten in pulse energy and close to 100 in harmonic power. We stress again that the optimum configuration for harmonic generation depends entirely on the particular application the harmonics are designed for.

Finally, we use the GA to identify configurations such that a train of attosecond pulses is obtained in the time profile of the XUV radiation. In analogy with laser mode locking, the time structure of the combination of several phase-locked harmonics consists of a train of ultra-short pulses [26]. The phase locking of the harmonics depends on the relative contributions of the different quantum paths discussed above. If several quantum paths contribute to the dipole moment with comparable amplitudes, adjacent harmonics are not phase locked due to the resulting interference, and the time structure of the XUV radiation over one half laser period is complex with several sub-structures. If in contrast only one quantum path dominates, the harmonics do phase lock, which leads to a time profile consisting of one attosecond pulse every half cycle of the laser field [26]. 


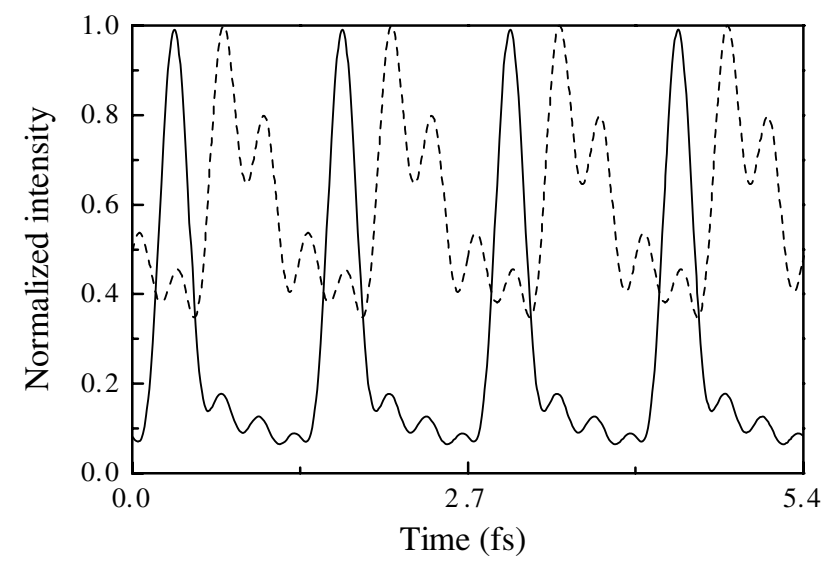

Figure 6. Time profile of the harmonics $15-23$ at an intensity of $2.4 \times 10^{14} \mathrm{~W} \mathrm{~cm}^{-2}$, in the conditions where the temporal coherence of the 19th harmonic is optimized (solid curve). The dashed curve shows the corresponding time profile in the conditions from 3.1.2.

To identify such conditions, we consider the 19th harmonic in argon at an intensity of $2.4 \times 10^{14} \mathrm{~W} \mathrm{~cm}^{-2}$, where the harmonic is well in the plateau region of the harmonic spectrum. We then optimize its temporal coherence with respect to $L$ and $Z_{0}$ as described above. We check that the optimum configuration also leads to narrow spectra for all neighbouring harmonics, from the 15 th to the $23 \mathrm{rd}$. In figure 6 we show the time profile $I_{\mathrm{XUV}}\left(t^{\prime}\right)$ of the XUV radiation close to the peak of the laser pulse $\left(t_{0}=0\right)$, obtained in the optimum configuration. It is given by

$$
I_{\mathrm{XUV}}\left(t^{\prime}\right)=\int\left|\sum_{q=N}^{q=M} E_{q}\left(r, t_{0}\right) \exp \left(-\mathrm{i} q \omega_{0} t^{\prime}\right)\right|^{2} 2 \pi r \mathrm{~d} r
$$

when including the harmonics $N-M$, and $\omega_{0}$ is the laser frequency. It presents a very nice train of attosecond pulses, consisting of one large burst of XUV radiation with an FWHM of 265 as, and a central frequency of $29 \mathrm{eV}$, every half cycle of the laser field. For comparison, the dashed curve shows the time profile in the configuration from 3.1.2 where the 15th harmonic pulse energy was optimized.

\section{Summary}

We have used a GA to optimize in turn the yield, the pulse duration and the temporal coherence of harmonic pulses. We find that these properties are optimized in very different configurations. A search algorithm can thus be very useful in identifying favourable conditions for a particular application of the harmonic radiation.

We have obtained up to $10^{9}$ photons per pulse for both plateau and cutoff harmonics in argon, and pulse durations as low as 6 fs generated by 50 fs laser pulses. The harmonic generation and phase matching processes are complicated and influenced by a number of factors, and the optimum configuration for each harmonic is therefore very sensitive to the fixed initial conditions. However, we find that different harmonics in the plateau region of the harmonic spectrum behave similarly to each other, and likewise for the cutoff harmonics, so that an optimization of the 15th harmonic for instance also yields good conditions for the 17 th harmonic. 
We used this fact to find conditions where part of the XUV radiation is emitted as a train of attosecond pulses. Since the configuration which optimizes the temporal coherence of the 19th harmonic also leads to good temporal coherence of the neighbouring harmonics, the combination of the harmonics 15-23 presents the desired time structure. We could successfully optimize the temporal coherence of the plateau harmonics in argon by tailoring the phase matching conditions to spectrally select the well behaved part of the XUV radiation. Experimentally, harmonic spectra are relatively easy to measure and characterize compared with harmonic time profiles. We have shown in this work that by using an algorithm to optimize spectral properties, it is possible to identify not only temporally coherent XUV radiation, but even conditions for generating attosecond pulse trains.

\section{Acknowledgments}

We gratefully acknowledge support from the Swedish Natural Science Research Council and from the Göran Gustafsson Foundation for Research in Natural Science and Medicine. LR is also grateful for the travel support provided by the Royal Physiographical Society in Sweden. Computer time was in part provided by the National Supercomputer Centre in Sweden. The authors also thank J Krause and K Schafer for stimulating discussions.

\section{References}

[1] Chang Z, Rundquist A, Wang H, Murnane M M and Kapteyn H C 1997 Phys. Rev. Lett. 792967

[2] Spielmann Ch, Burnett N H, Sartania S, Koppitsch R, Schnürer M, Kan C, Lenzner M, Wobrauschek P and Krausz F 1997 Science 278661

[3] Gisselbrecht M, Descamps D, Lyngå C, L'Huillier A, Wahlström C-G and Meyer M 1999 Phys. Rev. Lett. 82 4607

[4] Haight R and Peale D R 1993 Phys. Rev. Lett. 703979

[5] Descamps D, Lyngå C, Norin J, L'Huillier A, Wahlström C-G, Hergott J-F, Merdji H, Salières P, Bellini M and Hänsch T W 2000 Opt. Lett. 25135

[6] Sekikawa T, Ohno T, Yamazaki T, Nabekawa Y and Watanabe S 1999 Phys. Rev. Lett. 832564

[7] Descamps D, Roos L, Delfin C, L'Huillier A and Wahlström C-G 2001 Phys. Rev. A 64 031404(R)

[8] Paul P M, Breger P, Agostini P, Toma E S, Muller H G, Mullot G, Augé F and Balcou P 2001 Science 2921689

[9] Bouhal A, Salières P, Breger P, Agostini P, Hamoniaux G, Mysyrowicz A, Antonetti A, Constantinescu R and Muller H G 1998 Phys. Rev. A 58389

[10] Sheehy B et al 1999 Phys. Rev. Lett. 835270

[11] de Bohan A, Antoine Ph, Milosević D B and Pireaux B 1998 Phys. Rev. Lett. 811837

[12] Schafer K J and Kulander K C 1997 Phys. Rev. Lett. 78638

[13] Bartels R, Backus S, Zeek E, Misoguti L, Vdovin G, Christov I P, Murnane M M and Kapteyn H C 2000 Nature 406164

[14] Salières P, L'Huillier A and Lewenstein M 1995 Phys. Rev. Lett. 743776

[15] Lyngå C, L'Huillier A and Wahlström C-G 1996 J. Phys. B: At. Mol. Opt. Phys. 293293

[16] Altucci C, Starczewski T, Mevel E, Wahlström C-G, Carré B and L'Huillier A 1996 J. Opt. Soc. Am. B 13148

[17] Constant E, Garzella D, Berger P, Mével E, Dorrer Ch, Le Blanc C, Salin F and Agostini P 1999 Phys. Rev. Lett. 821668

[18] Delfin C, Altucci C, De Filippo F, de Lisio C, Gaarde M B, L'Huillier A, Roos L and Wahlström C-G 1999 J. Phys. B: At. Mol. Opt. Phys. 325397

[19] Goldberg D E 1989 Genetic Algorithms in Search, Optimization and Machine Learning (Reading, MA: AddisonWesley)

[20] Holland J H 1992 Sci. Am. 267 44-50

[21] Baumert T, Brixner T, Seyfried V, Strehle M and Gerber G 1997 Appl. Phys. B 65779

[22] Christov I P, Bartels R, Kapteyn H C and Murnane M M 2001 Phys. Rev. Lett. 865458

[23] Bellini M, Lyngå C, Tossi A, Gaarde M B, Hänsch T W, L'Huillier A and Wahlström C-G 1998 Phys. Rev. Lett. 81297 
[24] Gaarde M B, Salin F, Constant E, Balcou Ph, Schafer K J, Kulander K C and L’Huillier A 1999 Phys. Rev. A 59 1367

[25] Salières P, Carré B, Le Déroff L, Grasbon F, Paulus G G, Walther H, Kopold R, Becker W, Miloševic D B, Sanpera A and Lewenstein M 2001 Science 292902

[26] Antoine Ph, L'Huillier A and Lewenstein M 1996 Phys. Rev. Lett. 771234

[27] Lewenstein M, Balcou Ph, Ivanov M Yu, L'Huillier A and Corkum P B 1994 Phys. Rev. A 492117

[28] Kulander K C, Schafer K J and Krause J L 1992 Atoms in Intense Radiation Fields ed M Gavrila (New York: Academic)

[29] Antoine Ph, L'Huillier A, Lewenstein M, Salières P and Carré B 1996 Phys. Rev. A 531725

[30] Ilkov F A, Decker J E and Chin S L 1992 J. Phys. B: At. Mol. Opt. Phys. 254005

[31] We use the GENESIS 5.0 software package written by John J Grefenstette: 1989 GENESIS: a system for using genetic search procedures Proc. 1984 Conf. on Genetic Algorithms pp 70-9 (San Mateo, CA: Morgan Kaufmann) available on the internet from http://www.aic.nrl.navy.mil/galist/src/

[32] Lewenstein M, Salières P and L'Huillier A 1995 Phys. Rev. A 524747 